\title{
Scale Space Analysis and Active Contours for Omnidirectional Images
}

\author{
Iva Bogdanova ${ }^{1,2}$, Xavier Bresson ${ }^{1}$, Jean-Philippe Thiran $^{1}$ and Pierre Vandergheynst ${ }^{1}$
}

\begin{abstract}
A new generation of optical devices that generate images covering a larger part of the field of view than conventional cameras, namely catadioptric cameras, is slowly emerging. These omnidirectional images will most probably deeply impact computer vision in the forthcoming years, providing the necessary algorithmic background stands strong. In this paper we propose a general framework that helps defining various computer vision primitives. We show that geometry, which plays a central role in the formation of omnidirectional images, must be carefully taken into account while performing such simple tasks as smoothing or edge detection. Partial Differential Equations (PDEs) offer a very versatile tool that is well suited to cope with geometrical constraints. We derive new energy functionals and PDEs for segmenting images obtained from catadioptric cameras and show that they can be implemented robustly using classical finite difference schemes. Various experimental results illustrate the potential of these new methods on both synthetic and natural images.
\end{abstract}

\section{INTRODUCTION}

Conventional imaging systems are severely limited in their fi eld of view so that, in order to obtain an image of an entire scene, either multiple or rotating cameras must be used. But in many cases a rotating camera is not suitable because it can not simultaneously cover all directions of a dynamic scene. On the other hand mechanical parts are usually heavy and expensive to manufacture. An interesting alternative way to enhance the fi eld of view is to use mirrors in conjunction with lenses. A catadioptric sensor is an imaging sensor based on the combination of a curved mirror and a lens to form a projection onto the image plane of a camera (see Figure 1). Such a sensor is capable of recording light rays coming from a

\footnotetext{
I.B. and P.V. acknowledge the support of the Swiss National Science Foundation through grant FNS 200021-101880/1

${ }^{1}$ Insitute of Microtechnology, Université de Neuchâtel, 2000 Neuchâtel, Switzerland. E-mail: iva.bogdanova@unine.ch

${ }^{2}$ Signal Processing Institute, Ecole Polytechnique Fédérale de Lausanne (EPFL), 1015 Lausanne, Switzerland. E-mail: \{xavier.bresson, jean-philippe.thiran, pierre.vandergheynst $\} @$ epfl.ch
} 
broad range of directions around the focal point of the mirror. Consequently the images obtained from these sensors are often called omnidirectional because they contain the information from a scene in all possible directions around a perfect point-wise observer.

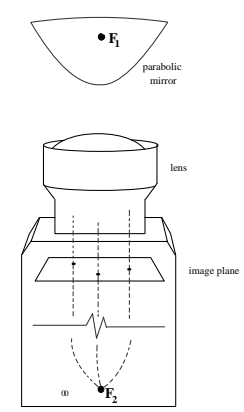

Fig. 1. A catadioptric imaging system.

Various mirror shapes can be used but it has been shown in [1] that the cases of interest are spherical, hyperbolical and parabolical mirrors, among which only the last two have a single effective viewpoint. Examples of images acquired using catadioptric cameras are provided on Figure 2.

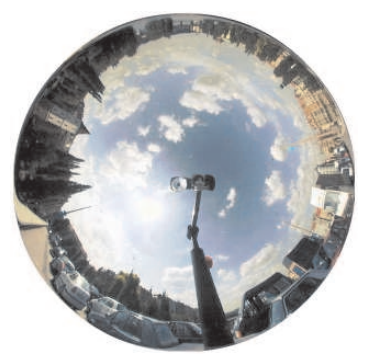

(a)

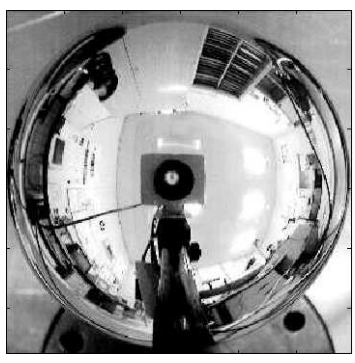

(b)

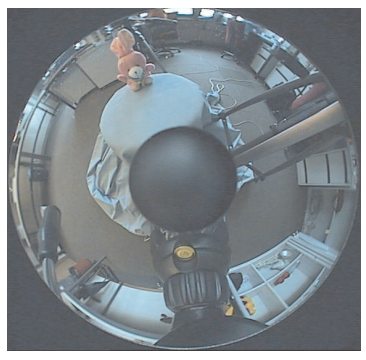

(c)

Fig. 2. Examples of omnidirectional images obtained using different mirrors: (a) hyperbolic, (b) spherical, (c) parabolic.

Obviously the sensor records a distorted image. The formation of the omnidirectional image is wellcontrolled and allows us to easily derive its geometrical properties. First the mirror is a surface $\mathcal{M}$ in $\mathbb{R}^{3}$ whose geometrical properties are encoded in its induced Riemannian metric $g_{i j}$. Second, and since we are considering perfect quadrics only, any light ray incident on one of the foci of the mirror is reflected to the other focus. A pixel is then created where the reflected light ray intersects the camera plane. Let $(x, y)$ and $\left(x_{0}, x_{1}, x_{2}\right)$ label coordinates in the image plane, here an open subset $\Omega \subseteq \mathbb{R}^{2}$, and on 
the mirror surface respectively. The whole image formation process depicted above induces a mapping between manifolds from the surface of the mirror to the camera plane :

$$
\begin{aligned}
\Phi: \mathcal{M} & \mapsto \Omega \\
\left(x_{0}, x_{1}, x_{2}\right) & \mapsto(x, y) .
\end{aligned}
$$

This application allows us to transport the metric $g_{i j}\left(x_{0}, x_{1}, x_{2}\right)$ and equip the image with a pullback metric $h_{i j}(x, y)$ :

$$
h_{j l}=\frac{\partial}{\partial u_{j}} x_{r} \frac{\partial}{\partial u_{l}} x_{s} g_{r s}, \quad j, l \in\{1,2\}, r, s \in\{0,1,2\},
$$

where we set $u_{1}=x, u_{2}=y$ and Einstein's summation convention has been used. Two comments are in order here. First, it should be noted that the image plane $(\Omega, h)$ shouldn't be dealt with as the familiar Euclidean plane. It carries a very specifi c geometry encoded in its metric $h_{i j}$. Second, this geometry is really inherited from that of the mirror $(\mathcal{M}, g)$, but realized using a more convential domain $\Omega \subseteq \mathbb{R}^{2}$. This simple, yet geometrically accurate, parameterization will be most helpful to perform computation on $\mathcal{M}$. Besides $(\mathcal{M}, g)$ and $(\Omega, h)$ there is another manifold of interest for omnidirectional images: the two-dimensional sphere $S^{2}$ in $\mathbb{R}^{3}$. It is indeed very interesting to be able to reconstruct the whole visual information around a perfect point-like observer standing at the focus of the mirror. In this case, each point $(\theta, \varphi) \in S^{2}$ represents the direction of an incoming light ray. For simple mirrors it is possible to reconstruct this information directly from the omnidirectional image in the sensor plane $\Omega$.

As we shall see throughout this paper, the geometry embodied by the metrics $g$ or $h$ is of primal importance. We will thus treat omnidirectional images as scalar fi elds on parametric manifolds. Geometry, and more particularly the Riemannian structure, will be used to derive generalizations of the concepts of smoothing, scale-space and geodesic active contours for this particular kind of images. We will proceed by deriving a fairly general framework based on energy minimization and partial differential equations (PDEs). Moreover we will make use of the parameterization to compute effi ciently these operations directly from the camera plane. These techniques are of course directly applicable to situations where scalar data are drawn on a parametric surface $\mathcal{M}$ for which we have a decent projection to a euclidean parametric base $\Omega$. In these cases, the framework derived in this paper allows to effi ciently implement the above-mentioned algorithms directly in the parametric cartesian coordinates, with well-studied numerical schemes, while still respecting the complex geometry of the manifold.

This paper is organized as follows. In Section II we review the geometry of the problem, particularizing to catadioptric systems. In Section III we introduce basic differential operators on Riemannian manifolds 
and provide explicit formulas for the surfaces of interest in this paper. Section IV is the core of the paper, where we construct active contours on parametric surfaces. The case of surfaces conformally euclidean is solved by a straightforward extension of the technique originally introduced in [2]. For more complex situations, we formulate a new energy minimization problem inspired by the formalism introduced in [3]. We illustrate our results with numerical simulations using omnidirectional images but also on generic situation where data is defi ned on surfaces. Finally, Section V gathers conclusions and suggestions for future research.

\section{RIEMANNIAN GEOMETRY OF PARAMETRIC SURFACES}

In this section, we introduce few concepts of Riemannian geometry that will be used throughout this paper. This material is classical and can be found in standard textbooks (see for example [4]), but we include it here for clarity and as a way to introduce notations. These notions are first worked out in the case of omnidirectional images produced with parabolic catadioptric mirrors, which will be our main application. Since the techniques designed in this paper are fairly general, we also give explicit derivations in the case of scalar images drawn on the surface of a hyperboloid. These two case-studies will be used later on in the paper in a recurrent way.

\section{A. Parabolic catadioptric mirrors}

A complete geometrical view of catadioptric systems has been worked out in [1], to which we refer for details. That paper shows that the most interesting catadioptric systems use either a parabolic or hyperbolic mirror. We will deal here with parabolic mirrors only, as they are by far the most common. None of our results are strictly bound to this particular case, though. In fact the techniques introduced below could be used for any mirror represented as a parametric surface.

The paraboloid $\mathcal{P}^{2}$ is a quadratic surface which can be expressed by the Cartesian equation

$$
x_{0}=b\left(x_{1}^{2}+x_{2}^{2}\right) .
$$

We will set $b=1$ for simplicity. Using a system of polar coordinates $(r, \varphi)$ shown on Figure 3 we obtain the equivalent parameterization :

$$
\begin{aligned}
& x_{0}=r^{2} \\
& x_{1}=r \cos \varphi, \quad 0 \leq \varphi<2 \pi, \quad r \geq 0 \\
& x_{2}=r \sin \varphi .
\end{aligned}
$$



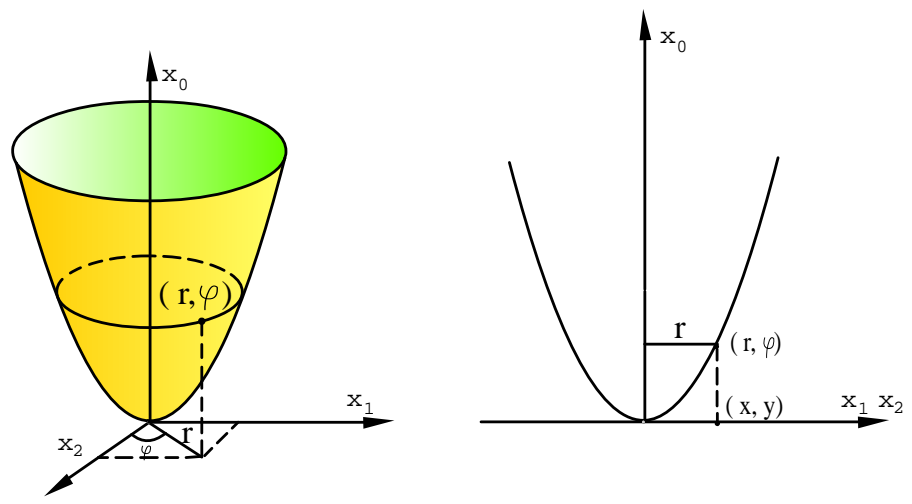

Fig. 3. Geometry of the paraboloid $\mathcal{P}^{2}$ embedded in $\mathbb{R}^{3}$. Left: The original coordinates $\left(x_{0}, x_{1}, x_{2}\right)$ expressed in polar coordinates $(r, \varphi)$ are mapped to coordinates $(x, y)$ after focal projection. Right: The focal projection coincides with a simple orthographic projection in this case.

The Euclidean line element can be expressed in these coordinates:

$$
\mathrm{d} l^{2}=\mathrm{d} x_{0}^{2}+\mathrm{d} x_{1}^{2}+\mathrm{d} x_{2}^{2}=\left(1+4 r^{2}\right) \mathrm{d} r^{2}+r^{2} \mathrm{~d} \varphi^{2},
$$

and this immediately gives us the metric on $\mathcal{P}^{2}$ :

$$
g_{i j}(r, \varphi)=\left(\begin{array}{cc}
1+4 r^{2} & 0 \\
0 & r^{2}
\end{array}\right) .
$$

As shown by Geyer and Daniilidis in [1], any light ray incident to the focus of the paraboloid is going to be reflected to the other focus, here the point at infi nity. Thus the focal projection $\Phi$ from the mirror $\mathcal{M}$ to the camera plane is simply the orthographic projection, i.e. the coordinates of the intersection between the reflected light ray and the camera plane are simply $(x, y)=\left(x_{1}, x_{2}\right)$. In the following we shall need the induced metric on the camera plane in $(x, y)$ coordinates. Since we have $r^{2}=x^{2}+y^{2}$ and $\varphi=\arctan \frac{y}{x}$, performing this change of variable in the 2-D Euclidean line element yields :

$$
\mathrm{d} l^{2}=\left(1+4 x^{2}\right) \mathrm{d} x^{2}+8 x y \mathrm{~d} x \mathrm{~d} y+\left(1+4 y^{2}\right) \mathrm{d} y^{2},
$$

which corresponds to the following metric:

$$
h_{i j}(x, y)=\left(\begin{array}{cc}
1+4 x^{2} & 4 x y \\
4 x y & 1+4 y^{2}
\end{array}\right) .
$$

Note that this metric is not diagonal; it will be an important source of complication later on.

In the case of a parabolic mirror, Geyer and Daniilidis showed that the reconstruction of a perfect viewpoint at the focus of the mirror can be simply computed as the inverse stereographic projection of 
the sensor image. It is thus also important to work out the links between the geometry of $S^{2}$ and that of the sensor image. As we shall see in the next sections, this will allow us to process the spherical image directly using the sensor output. Consider a sphere of radius $r$ as depicted on Figure 4. A point on $S^{2}$ is identified with the vector $\left(x_{0}, x_{1}, x_{2}\right) \equiv(r \cos \theta, r \sin \theta \sin \varphi, r \sin \theta \cos \varphi), \theta \in[0, \pi), \varphi \in[0,2 \pi)$. In cartesian and polar $(\rho, \theta, \varphi)$ coordinates, the Euclidean line element reads :

$$
\mathrm{d} l^{2}=\mathrm{d} x_{0}^{2}+\mathrm{d} x_{1}^{2}+\mathrm{d} x_{2}^{2}=\mathrm{d} \rho^{2}+\rho^{2}\left(\mathrm{~d} \theta^{2}+\sin ^{2} \theta \mathrm{d} \varphi^{2}\right) .
$$

On the surface $\rho=r$ and the differential $\mathrm{d} \rho=0$, so the metric induced on the sphere is given by the well-known expression :

$$
\mathrm{d} l^{2}=r^{2}\left(\mathrm{~d} \theta^{2}+\sin ^{2} \theta \mathrm{d} \varphi^{2}\right)
$$

The stereographic projection sends a point $(\theta, \varphi)$ on the sphere to the point with polar coordinates $(R, \varphi)$ in the plane, for which we have $\varphi=\varphi, R=r \frac{1}{2} \tan \theta / 2$. It is shown on Figure 4. In terms of these new coordinates the metric becomes

$$
\mathrm{d} l^{2}=\frac{4 r^{4}}{\left(r^{2}+R^{2}\right)^{2}}\left(\mathrm{~d} R^{2}+R^{2} \mathrm{~d} \varphi^{2}\right)
$$

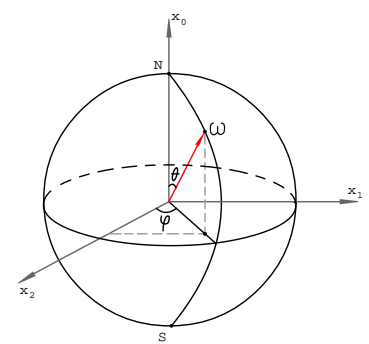

(a)

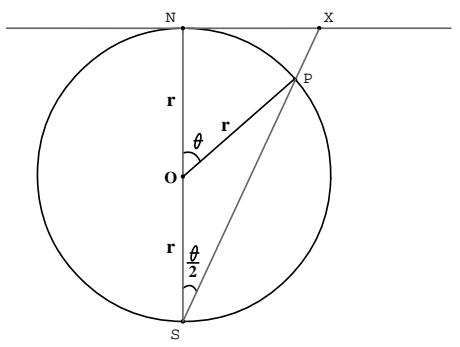

(b)

Fig. 4. Geometry of the 2-sphere: (a) spherical polar coordinates (b) stereographic projection.

Let us proceed toward deriving the metric in cartesian sensor coordinates $(x, y) \in \mathbb{R}^{2}$, where $R^{2}=$ $x^{2}+y^{2}$. We obtain

$$
\mathrm{d} l^{2}=\frac{4 r^{4}}{\left(r^{2}+x^{2}+y^{2}\right)^{2}}\left(\mathrm{~d} x^{2}+\mathrm{d} y^{2}\right) .
$$

In this case the metric on the sphere is obtained from the metric on the Euclidean plane by multiplying the latter by the function $\frac{4 r^{2}}{\left(r^{2}+x^{2}+y^{2}\right)^{2}}$ :

$$
\mathrm{d} l_{S^{2}}^{2}=\frac{4 r^{4}}{\left(r^{2}+x^{2}+y^{2}\right)^{2}} \mathrm{~d} l_{\mathbb{R}^{2}}^{2} .
$$


Accordingly, the metric induced on $\mathbb{R}^{2}$ when $R=1$ is derived as

$$
h_{i j}(x, y)=\left(\begin{array}{cc}
\frac{4}{\left(1+x^{2}+y^{2}\right)^{2}} & 0 \\
0 & \frac{4}{\left(1+x^{2}+y^{2}\right)^{2}}
\end{array}\right),
$$

and consequently the inverse metric is as follows:

$$
h^{i j}(x, y)=\left(\begin{array}{cc}
\frac{\left(1+x^{2}+y^{2}\right)^{2}}{4} & 0 \\
0 & \frac{\left(1+x^{2}+y^{2}\right)^{2}}{4}
\end{array}\right) .
$$

Metrics that differ only by a multiplicative factor are conformally equivalent. The stereographic projection endows the plane with a metric conformal to the regular euclidean metric. This particular class of problems will receive special attention later on in this paper. Finally, one shouldn't be surprised that metrics (8) and (14) are so different. Though the related images are produced by the same system, the first one corresponds to light intensity on the parabolic mirror while the second one corresponds to light intensity perceived by an observer at the focus of the mirror. The same sensor image is thus endowed with different geometries, depending on which information one wishes to process.

\section{B. Another illustrative example}

Let us now give another illustrative example : a different parametric surface, together with a projection to a parametric base. Consider the two-sheeted hyperboloid $H^{2}$ depicted on Figure 5. It is convenient to embed $H^{2}$ into the pseudo-Euclidean (or Minkowski) space $\mathbb{R}_{1}^{2}$, whose line element is

$$
\mathrm{d} l^{2}=\mathrm{d} x_{0}^{2}-\mathrm{d} x_{1}^{2}-\mathrm{d} x_{2}^{2} .
$$

In these coordinates, $H^{2}$ is conveniently realized a pseudo-sphere of equation :

$$
x_{0}^{2}-x_{1}^{2}-x_{2}^{2}=R^{2} .
$$

In pseudo-polar coordinates $\left(x_{0}, x_{1}, x_{2}\right) \equiv(\rho \cosh \chi, \rho \sinh \chi \cos \varphi, \rho \sinh \chi \sin \varphi)$, equation (16) becomes :

$$
\mathrm{d} l^{2}=-\rho^{2}\left(\mathrm{~d} \chi^{2}+\sinh ^{2} \chi \mathrm{d} \varphi^{2}\right)+\mathrm{d} \rho^{2} .
$$

For $\rho=R$ we distinguish the upper sheet of the hyperboloid, and for $\rho=-R$, the lower one. Since $\rho$ is constant on the hyperboloid, we fi nally have

$$
-\mathrm{d} l^{2}=R^{2}\left(\mathrm{~d} \chi^{2}+\sinh ^{2} \chi \mathrm{d} \varphi^{2}\right)
$$

and the metric on the upper sheet of $H^{2}$ reads

$$
g_{i j}(\chi, \varphi)=\left(\begin{array}{cc}
1 & 0 \\
0 & \sinh ^{2} \chi
\end{array}\right),
$$




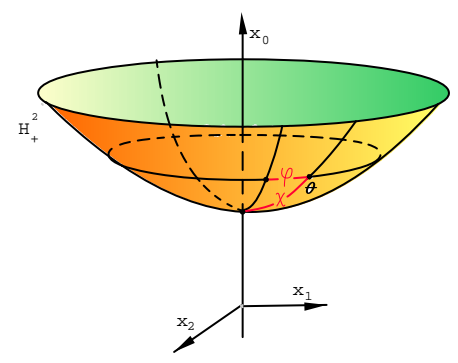

(a)

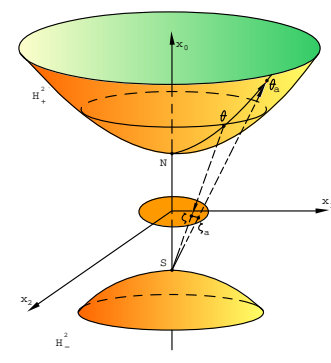

(b)

Fig. 5. Geometry of the 2-hyperboloid: (a) hyperbolic polar coordinates (b) stereographic projection.

where we set $R=1$.

A useful parametric realization of the upper sheet of the hyperboloid consists in projecting inside the open unit disk in $\mathbb{R}^{2}$ by means of a stereographic projection (see Figure 5) :

$$
\begin{aligned}
& x=\tanh \frac{\chi}{2} \cos \varphi \\
& y=\tanh \frac{\chi}{2} \sin \varphi .
\end{aligned}
$$

Note that if the eccentricity of the hyperboloid is big enough, this mapping closely matches the focal projection of a hyperbolic mirror and can be used for omnidirectional image processing as well. The induced metric in the disk is easily computed :

$$
h_{i j}(x, y)=\left(\begin{array}{cc}
\frac{4}{\left(1-\left(x^{2}+y^{2}\right)\right)^{2}} & 0 \\
0 & \frac{4}{\left(1-\left(x^{2}+y^{2}\right)\right)^{2}}
\end{array}\right) .
$$

Note the formal similarities with the expressions derived above for the sphere and the general form of this metric, which is also conformally equivalent to the euclidean one.

Before concluding this section, we may wonder why one would work on the manifold-bound data instead of simply considering the acquired image as a regular euclidean one. We do believe there are simple tasks that can be performed directly on the sensor image, without resorting to the complex framework discussed hereafter. But there are important situations where working on the manifold is of vital importance. For example, it has been shown in [5] that working with a full fi eld of view (a perfect omnidirectional image) stabilizes the structure from motion problem and that the reason for this gain is purely geometrical. Working with the image as observed by an observer at the focus of the mirror has also numerous advantages for tracking, since it processes the fi eld of view in its natural coordinates. Finally, using the correct geometry is also of paramount importance in pattern recognition : on the sensor output seen as regular euclidean image, objects of the same size or shape will appear distorted depending 
on their position, whereas these effects are nicely compensated for in the correct geometry. We will give a concrete example example of these effects in Section IV-C.

\section{SMOOTHING AND DETECTING EDGES ON PARAMETRIC MANIFOLDS}

\section{A. Warm-up: useful differential operators}

Various classical tasks in Computer Vision (smoothing, edge detection) can be implemented by means of differential operators acting on images. Since we have defi ned omnidirectional images as scalar functions on Riemannian manifolds, we can now easily defi ne the corresponding differential operators in the correct geometry. Even though most of this material can be found in standard textbooks ([4]), we provide here the most important expressions for completeness. One of the fi rst differential operators encountered in image processing is the gradient, used primarily for edge detection. The gradient of a scalar function $I$ is defi ned in a coordinate-free fashion in $\mathbb{R}^{n}$ by imposing that the directional derivative of a scalar fi eld satisfi es :

$$
D_{\mathbf{v}} I=\nabla I \cdot \mathbf{v}
$$

On a Riemannian manifold $\left(\mathcal{M}, g_{i j}\right)$, directional derivatives are replaced by vectors $\mathbf{v}$ in the tangent plane of $\mathcal{M}$ at a point $p$ and the scalar product at $p$ is naturally defi ned through the metric :

$$
\mathbf{v}[I]=\langle\nabla I, \mathbf{v}\rangle_{p}
$$

In a local system of coordinates $x^{i}$ on $\mathcal{M}$, the components of the gradient will thus read :

$$
\nabla^{i}=g^{i j} \frac{\partial}{\partial x^{j}}
$$

A similar reasoning leads to the expression of the divergence of a vector fi eld $\mathbf{X}$ on $\mathcal{M}$ :

$$
\operatorname{div} \mathbf{X}=\frac{1}{\sqrt{g}} \partial_{i}\left(X^{i} \sqrt{g}\right)
$$

where $g$ is the determinant of $g^{i j}$. Finally, just as the ordinary Laplacian is defi ned in $\mathbb{R}^{n}$ by combining these two operators, the Laplace-Beltrami [6] operator is the second order differential operator defi ned on scalar fi elds on $\mathcal{M}$ by :

$$
\Delta I=-\frac{1}{\sqrt{g}} \partial_{j}\left(g^{i j} \sqrt{g} \partial_{i} I\right)
$$




\section{B. Examples}

As a first example of the concepts introduced above, let us consider the problem of detecting edges of an omnidirectional image produced by a hyperbolic mirror. As depicted in Section II-B, we approximate the catadioptric system by a stereographic projection and work directly in the open unit disk in $\mathbb{R}^{2}$. The main advantage as we will see is that we can implement most techniques directly in cartesian coordinates from the sensor output while respecting the geometry of the mirror. The induced hyperbolic metric in the disk has been computed in (21) for cartesian coordinates $(x, y)$. Using (22) and since this metric is conformally equivalent to the euclidean cartesian metric, we see that the hyperbolic gradient is just a scaled version of its usual euclidean cousin :

$$
\nabla_{D}=\frac{\left(1-\left(x^{2}+y^{2}\right)\right)^{2}}{4} \nabla_{\mathbb{R}^{2}}
$$

The scaling factor in this expression goes to zero on the border of the disk, which does not belong to the image domain. It is exactly the conformal factor in the hyperbolic metric and governs the size and morphology of patterns in the image vary. To illustrate it's effect, we apply the gradient to localize edges of an omnidirectional image acquired with a hyperbolic mirror in Figure 6. More precisely, we computed the horizontal and vertical components of the gradient, as well as its norm. Note that in the latter case, the correct scalar product has been used :

$$
\begin{aligned}
\left|\nabla_{D} I\right|^{2} & =h_{i, j} \nabla_{D}^{i} I \nabla_{D}^{j} I \\
& =\frac{\left(1-\left(x^{2}+y^{2}\right)\right)^{2}}{4}\left|\nabla_{\mathbb{R}^{2}} I\right|^{2} .
\end{aligned}
$$

As a second example we would like to illustrate how one can easily implement gaussian smoothing or, equivalently, linear scale-space [7], [8], [9]. We will keep this discussion general enough so that it can be applied to problems other than omnidirectional computer vision. There are two popular ways of computing gaussian fi ltering in euclidean computer vision : either using convolution with a gaussian kernel or by implementing the linear heat flow. On general manifolds though, the situation becomes trickier. First, gaussian fi ltering may simply not exist : on the paraboloid for example, there is no clear notion of Fourier analysis or convolution theorem. In fact, these mathematical constructions make sense on homogeneous spaces of Lie groups, such as the sphere or the hyperboloid. But even in these nicer cases, the situation is not so simple. Indeed, if fi ltering can be easily implemented on $S^{2}$ thanks to a spherical equivalent of the Fast Fourier Transform [10], it is not the case on $H^{2}$ : there is a convolution and a Fourier transform (the Helgason transform, see [11]), but there is no effi cient algorithm and naive 


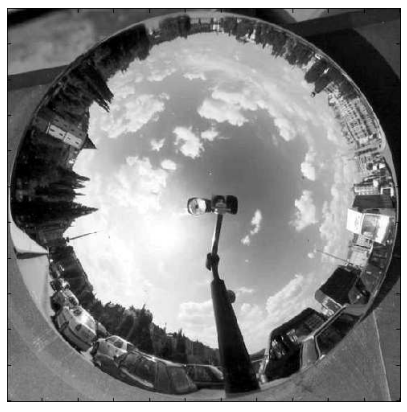

(a)

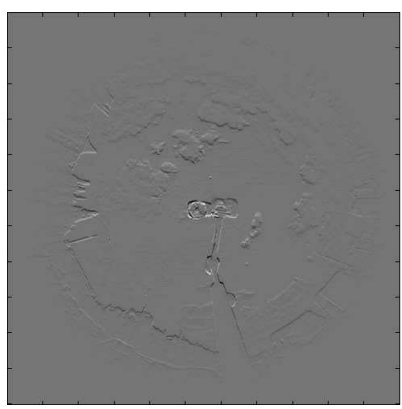

(c)

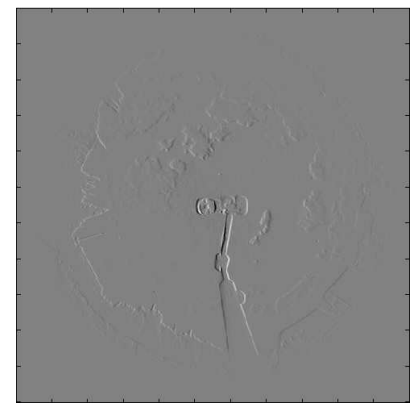

(b)

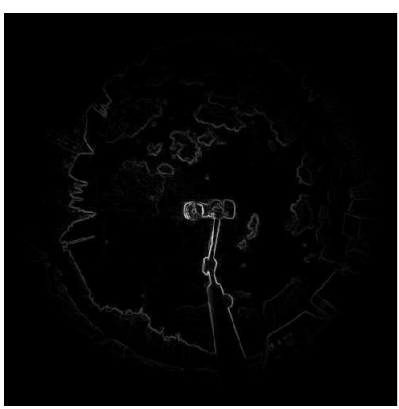

(d)

Fig. 6. Gradient of a hyperbolic image: (a) original image, (b) component of the gradient in the horizontal direction, (c) component of the gradient in the vertical direction, (d) norm of the gradient.

implementations based on separation of variables cannot be applied since the Helgason kernel is not separable. The best way to compute gaussian fi ltering on manifolds is thus to use the linear heat flow :

$$
\frac{\partial}{\partial t} I(\mathbf{x}, t)=\Delta I(\mathbf{x}, t)
$$

where $\Delta$ is the Laplace-Beltrami operator. Then again, implementing PDEs on manifolds may be quite complicated : usually differential operators are expressed in local coordinate systems which are hard to handle numerically.

In [12], [13], the authors introduced a new framework for solving variational problems and PDEs for scalar and and vector-valued data defi ned on arbitrary surfaces implicitly represented by a level set function. Level set functions are basically defi ned on a fi xed cartesian coordinate system, which allows easy computations of PDEs on level set-based surfaces contrary to surfaces represented by triangulated models. The main advantage of the previous framework is to carry out e.g. the linear heat flow on 
arbitrary surfaces even if the method requires to use a data extension procedure from the surface to a cartesian narrow band. It is however not clear what the exact extension procedure is. There are different models to extend data and one natural and fast method is to extend data in such a way that they are constant normal to each level set [14]. In our case of omnidirectional images, a global parametrization of surfaces is available, which allows to map the surface and its geometry to an euclidean parametric base. We thus propose to simply compute the heat flow on that base, which only requires cartesian differential operators, and then eventually map the result to the original surface. This approach provides fast and exact computations of the linear scale space without using a level set function or a triangulated surface but is limited to parametric surfaces.

Let us once again illustrate this mechanism with the hyperboloid. A scalar image painted on $H^{2}$ is fi rst mapped to the open unit disk by stereographic projection. The Escher painting on Figure 7 is a good example of such an image. The only thing we need to compute the heat flow, is the expression of the Laplace-Beltrami operator in the unit disk, given the metric (21):

$$
\begin{aligned}
\Delta_{D_{+}} I & =\frac{1}{\sqrt{h}}\left(\frac{\partial}{\partial_{x}} \sqrt{h} h^{x x} \frac{\partial I}{\partial x}+\frac{\partial}{\partial y} \sqrt{h} h^{y y} \frac{\partial I}{\partial y}\right) \\
& =\frac{\left(1-x^{2}-y^{2}\right)^{2}}{4}\left(\frac{\partial^{2} I}{\partial x^{2}}+\frac{\partial^{2} I}{\partial y^{2}}\right) \\
& =\frac{\left(1-x^{2}-y^{2}\right)^{2}}{4} \Delta_{\mathbb{R}^{2}} I .
\end{aligned}
$$

Once again, conformal equivalence has reduced this expression to a scaling factor times the usual Laplacian in $\mathbb{R}^{2}$. Implementation is thus trivial and requires only an effi cient discrete Laplacian [15]. The action of the Laplace-Beltrami heat flow in the disk is illustrated in Figure 7 for various evolution times $t$. As one can notice, the flow behaves like a linear euclidean heat flow only close to the origin. As one moves closer to the border of the disk, the scaling factor in (27) slows the flow down. This can also be understood intuitively as the size of patterns close to the border of the disk is actually much bigger than measured with euclidean tools. It will thus take more time to smooth them out of the image.

Deriving the gradient and Laplace-Beltrami operators was very simple in the case of $H^{2}$ thanks to conformal equivalence. The same of course holds true for the sphere. The case of the paraboloid is slightly more complicated but involves only straightforward applications of the defi nitions. For example, 


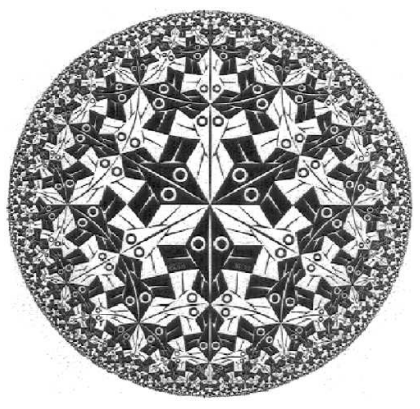

(a)

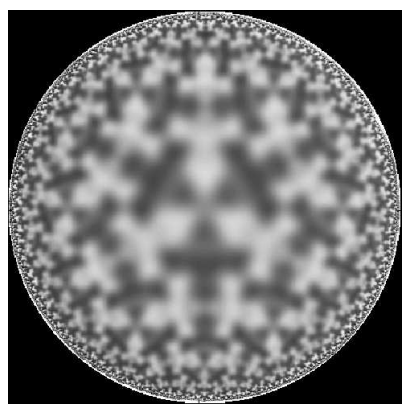

(d)

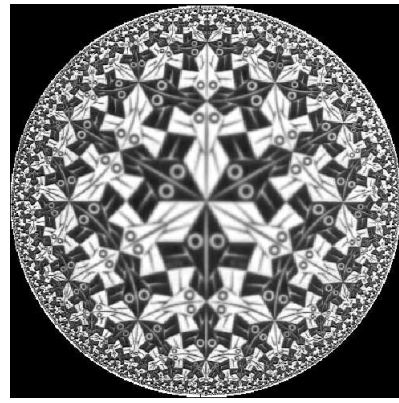

(b)

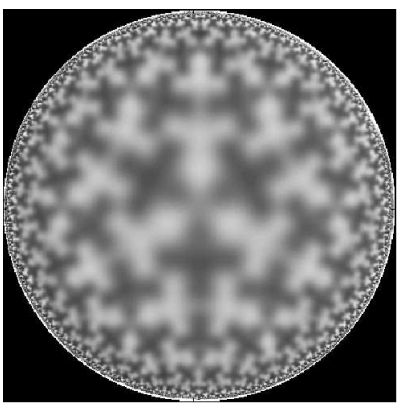

(e)

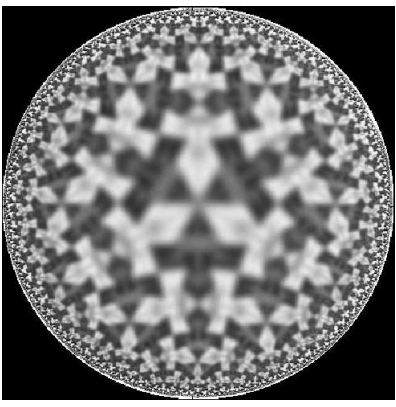

(c)

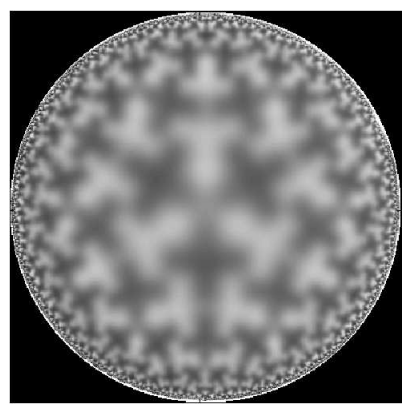

(f)

Fig. 7. Heat flow/Gaussian smoothing on a hyperbolic image: (a) original, (b) $t=10$, (c) $t=100$, (d) $t=300$, (e) $t=500$, (f) $t=700$. Note how the flow is comparatively slower close to the border of the disk.

the Laplace-Beltrami operator computed with the metric (8) reads :

$$
\begin{aligned}
\Delta I_{P^{2}}= & -\frac{1}{1+4 x^{2}+4 y^{2}} \times \\
& {\left[\left(1+4 y^{2}\right) \frac{\partial^{2} I}{\partial x^{2}}-4 x y \frac{\partial^{2} I}{\partial x \partial y}+\left(1+4 x^{2}\right) \frac{\partial^{2} I}{\partial y^{2}}\right.} \\
& \left.-\frac{8 x\left(1+2 x^{2}+2 y^{2}\right)}{\left(1+4 x^{2}+4 y^{2}\right)} \frac{\partial I}{\partial x}-\frac{8 y\left(1+2 x^{2}+2 y^{2}\right)}{\left(1+4 x^{2}+4 y^{2}\right)} \frac{\partial I}{\partial y}\right] .
\end{aligned}
$$

\section{Segmentation with Active Contours on Omnidirectional Images}

In this section, we defi ne the evolution equation of geodesic active contours tailored to omnidirectional images. We start by considering spherical and hyperbolic geometry, where conformal equivalence makes it easy to alter the initial theory. We then describe a broader technique that is able to cope with a larger class of manifolds, including the paraboloid. 


\section{A. Active Contour on Conformally Euclidean Surfaces}

Geodesic Active Contours (GAC) were introduced by Caselles-Kimmel-Sapiro in [2] and KichenassamyKumar-Olver-Tannenbaum-Yezzi in [16] to overcome the limitations of traditional snakes. They are defi ned as minimizers of following energy:

$$
\begin{aligned}
F_{G A C}(C) & =\int_{0}^{1} f\left(\left|\nabla I_{0}(C(p))\right|\right)\left|C_{p}\right| d p \\
& =\int_{0}^{L(C)} f\left(\left|\nabla I_{0}(C(s))\right|\right) d s
\end{aligned}
$$

where $C:[0,1] \mapsto \mathbb{R}^{2}$ is a closed planar curve, $C_{p}$ its fi rst derivative with respect to the parameter $p$, $L(C)$ is the Euclidean length of $C, I_{0}$ is a given image, represented here as a positive bounded function and $f$ is an edge detector function. It was proved in [2] that the direction for which $F_{G A C}$ decreases most rapidly provides the following minimization flow:

$$
\frac{\partial C}{\partial t}=(\kappa f-\langle\nabla f, \mathcal{N}\rangle) \mathcal{N}
$$

where $\mathcal{N}$ is the unit normal to the curve $C$ and $\kappa$ is its curvature. The right hand side of the equation (29) corresponds to the Euler-Lagrange of Energy (28). The fi rst term is the mean curvature motion, also called curve shortening flow, weighted by the edge detector function $f$. It smoothes the curve shape by decreasing its total length as fast as possible. The second term of (29) attracts the curve toward the boundaries of objects by creating an attraction valley centered on the edges. Hence, the function $f$ does not need to be equal to zero to stop the evolution of the snake on the contours of objects.

In [2], Caselles-Kimmel-Sapiro proved that the curve minimizing $F_{G A C}(C)$ is actually a geodesic in a Riemannian space whose metric tensor is:

$$
a_{i j}=f^{2}\left(\left|\nabla I_{0}\right|\right) \delta_{i j}
$$

where $\delta_{i j}$ is the Euclidean metric tensor. This fact is proved by considering the general defi nition of a geodesic, i.e. a curve of minimal weighted distance/length between two points on a given Riemannian space. The minimal length between two points on a manifold equipped with the metric tensor $a_{i j}$ is given by the following standard formula:

$$
\begin{aligned}
& \int_{0}^{1} \sqrt{a_{i j} C_{p}^{i} C_{p}^{j}} d p= \\
& \quad \int_{0}^{1} \sqrt{a_{11}\left(C_{p}^{1}\right)^{2}+2 a_{12} C_{p}^{1} C_{p}^{2}+a_{22}\left(C_{p}^{2}\right)^{2}} d p
\end{aligned}
$$


with $C=\left(C^{1}, C^{2}\right)$ and $C_{p}^{i}:=\frac{\partial C^{i}}{\partial p}$. Considering the metric (30), the active contour energy can be expressed in an equivalent way:

$$
\begin{aligned}
F_{G A C}(C) & =\int_{0}^{1} f\left(\left|\nabla I_{0}(C(p))\right|\right)\left|C_{p}\right| d p \\
& =\int_{0}^{1} f\left(\left|\nabla I_{0}(C(p))\right|\right) \sqrt{\delta_{i j} C_{p}^{i} C_{p}^{j}} d p \\
& =\int_{0}^{1} \sqrt{f^{2}\left(\left|\nabla I_{0}(C(p))\right|\right) \delta_{i j} C_{p}^{i} C_{p}^{j}} d p .
\end{aligned}
$$

This formalism encodes the geometry of edges in the new metric, but we see from (32) that the geometry of the evolving curve is still Euclidean. When the active contour $C$ evolves in a more general space, defined by the first fundamental form $h_{j}$, we thus propose to modify the formalism to take geometry into account. The most obvious modifi cation is to simply introduce the correct metric and this leads to a new energy functional :

$$
\int_{0}^{1} f\left(\left|\nabla I_{0}(C(p))\right|\right) \sqrt{h_{i j} C_{p}^{i} C_{p}^{j}} d p
$$

which reduces to the standard active contour model when the the metric $h_{i j}=\delta_{i j}$, i.e. the Euclidean metric.

Let us now go back to the specifi c case of omnidirectional images. The metric tensor for the sphere and the hyperboloid, defi ned in Equations (14) and (21), are both conformally equivalent to the euclidean metric through stereographic projection. This allows us to simplify Functional (33) considering $h_{i j}=$ $h^{1 / 2} \delta_{i j}$ where $h$ is the determinant of $h_{i j}$. Inserting this expression into (33), we get:

$$
\begin{gathered}
\int_{0}^{1} \underbrace{f\left(\left|\nabla I_{0}(C(p))\right|\right) h^{1 / 4}(C(p))}_{=: f_{D}(C(p))} \sqrt{\delta_{i j} C_{p}^{i} C_{p}^{j}} d p \\
=\int_{0}^{1} f_{D}(C(p))\left|C_{p}\right| d p=F_{A C S H M}(C),
\end{gathered}
$$

which corresponds to the energy of active contours on either spherical or hyperbolic manifolds ( $A C S H M)$ and $f_{D}$ (D stands for Disk) is the edge detector function for either the spherical or the hyperbolic (nonEuclidean) images. The evolution equation of the curve $C$ minimizing Energy (34) is naturally obtained by replacing the function $f$ by $f_{D}$ in (29):

$$
\begin{aligned}
\frac{\partial C}{\partial t} & =\left(\kappa f_{D}-\left\langle\nabla f_{D}, \mathcal{N}\right\rangle\right) \mathcal{N} \\
& =\left(\kappa f h^{1 / 4}-\left\langle\nabla\left(f h^{1 / 4}\right), \mathcal{N}\right\rangle\right) \mathcal{N} .
\end{aligned}
$$


We notice that Equation (36) is defi ned on the planar open domain $D$, which corresponds to the stereographic projection of the sphere and the hyperboloid onto the plane, see Sections II-A and II-B. Thus the evolution equation of the active contour is not directly performed on the spherical or the hyperbolic manifold but on the plane by taking into account the geometry of the given manifolds with the factor $h^{1 / 4}$. Then the snake is mapped onto the sphere and the hyperboloid with a one-to-one mapping defi ned in Sections II-A and II-B. This way of evolving the active contour on non-flat spaces, whose metric tensors are known, is thus very fast and numerically accurate. It does require a global parameterization and, so far, conformal equivalence with $\mathbb{R}^{2}$. In the next section, we will consider a larger class of manifolds.

Let us now consider two examples of illustration of these new active contour models. The fi rst example presents the evolution of the snake on the hyperbolic manifold by the mean curvature flow, Figure 8. The geometry of the hyperboloid is defi ned by the metric tensor in Equation (21), which implies that $h^{1 / 4}=\frac{2}{\left|1-\left(x^{2}+y^{2}\right)\right|}$. The mapping between the disk $D$, which corresponds to the stereographic projection of the hyperboloid onto the plane, is given by:

$$
\begin{aligned}
(x, y) \in D \rightarrow & \left(x_{H_{+}^{2}}, y_{H_{+}^{2}}, z_{H_{+}^{2}}\right)= \\
& (\sinh \chi \cos \varphi, \sinh \chi \sin \varphi, \cosh \chi) \in H_{+}^{2},
\end{aligned}
$$

given

$$
(\chi, \varphi)=\left(2 \tanh ^{-1}\left(\frac{1}{2} \sqrt{x^{2}+y^{2}}\right), \tan ^{-1}\left(\frac{y}{x}\right)\right) .
$$

The second example carries out the image segmentation task on the sphere by extracting Australia, Figure 11. The metric tensor of the sphere is defi ned in Equation (14), which implies that $h^{1 / 4}=$ $\frac{2}{\left|1+x^{2}+y^{2}\right|}$. Besides, the mapping between the plane $\Omega_{\theta, \varphi}$ of $(\theta, \varphi)$-values, given on Figure 10(a), and the disk $D$ on Figure 10(b), which corresponds to the stereographic projection of the sphere onto the plane, is given by:

$$
\begin{aligned}
(\theta, \varphi) \in \Omega_{\theta, \varphi} \rightarrow & (x, y)= \\
& \left(2 \tan \frac{\theta}{2} \cos \varphi, 2 \tan \frac{\theta}{2} \sin \varphi\right) \in D,
\end{aligned}
$$

and the mapping between the disk and the sphere $S^{2}$ is given by:

$$
\begin{aligned}
(x, y) \in D \rightarrow & \left(x_{S^{2}}, y_{S^{2}}, z_{S^{2}}\right)= \\
& (\cos \theta, \sin \theta \cos \varphi, \sin \theta \sin \varphi) \in S^{2},
\end{aligned}
$$




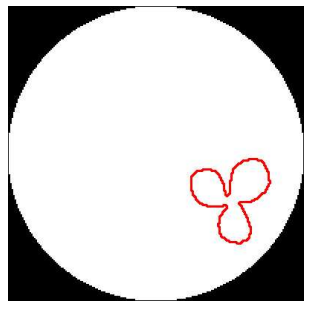

(a) Initial $\mathrm{AC}$ on

$D$

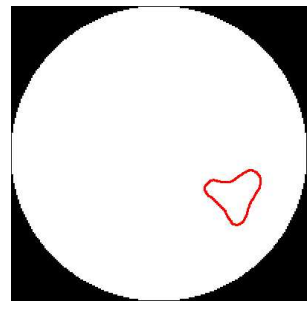

(c) $\mathrm{AC}$ at $t_{2}>t_{1}$

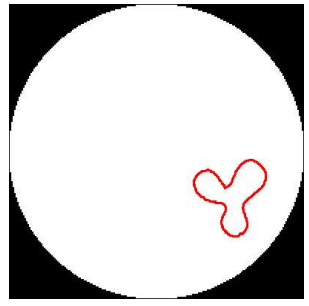

(b) $\mathrm{AC}$ at $t_{1}>0$

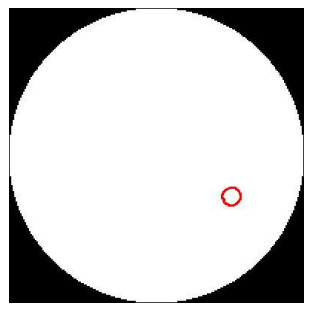

(d) $\mathrm{AC}$ at $t_{3}>t_{2}$

Fig. 8. Mean Curvature Flow on the Hyperboloid. Figures (a-d) present the evolution of the AC on the disk $D$ given by Equation (36) when $f=1$. The disk $D$ corresponds to the stereographic projection of the hyperboloid onto the plane.

given

$$
(\theta, \varphi)=\left(2 \tan ^{-1}\left(\frac{1}{2} \sqrt{x^{2}+y^{2}}\right), \tan ^{-1}\left(\frac{y}{x}\right)\right) .
$$

The numerical implementation of the minimizing flow (36) is done with classical numerical schemes, see [17], [18] for details.

\section{B. Active Contour on Parabolic Manifold}

In the previous section, an evolution equation for the active contours on spherical and hyperbolic manifolds was defi ned from the minimization of the functional (33) using the metric of these manifolds. These metrics have a simple form, $h_{i j}=h^{1 / 2} \delta_{i j}$, which allows us to directly find the evolution equation for the snake from the standard geodesic/geometric active contours [2], [16]. In this section, we want to derive the evolution equation for the active contours on parabolic manifolds whose metric tensor is defi ned in Equation (8). In this case, the metric is not diagonal, which implies more developments to fi nd the evolution equation from the minimization of (33). Instead of computing the Euler-Lagrange equation 


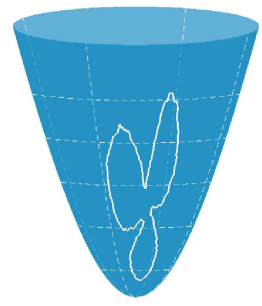

(a) Initial $\mathrm{AC}$ on $H_{+}^{2}$

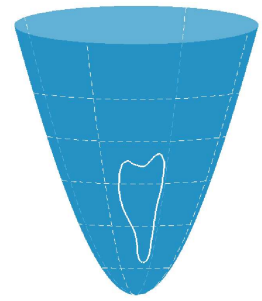

(c) AC at $t_{2}>$ $t_{1}$

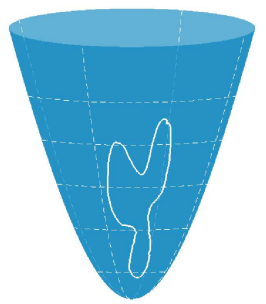

(b) AC at $t_{1}>$

0

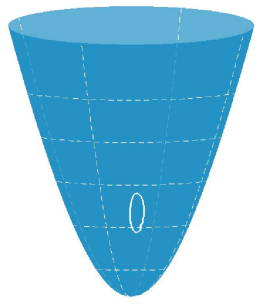

(d) $\mathrm{AC}$ at

$t_{3}>t_{2}$

Fig. 9. Mean Curvature Flow on the Hyperboloid. Figures (a-d) show the evolution of the AC of Figure 8 on the hyperboloid $H_{+}^{2}$ when using the mapping from the disk to the hyperbolid defi ned in Equation (37).
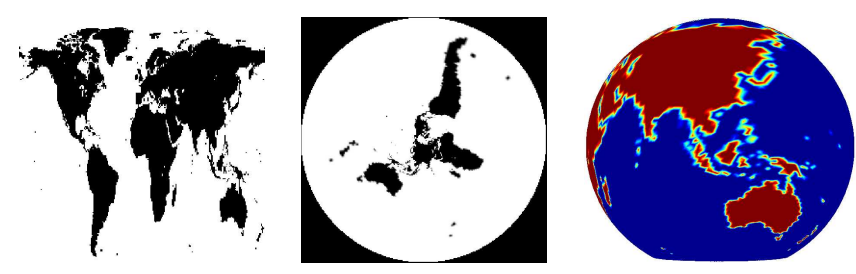

Fig. 10. Three equivalent representations of the omnidirectional image earth. (a) In the parametric $(\theta, \varphi)$ plane. (b) In the disk $D$ using the stereographic projection. (c) The original image as a scalar function on $S^{2}$.

of the functional (33), we propose to apply the formalism defi ned by Bresson-Vandergheynst-Thiran in [19], [3] which consider the general case of an active hypersurface evolving on any given Riemannian manifolds. This formalism is based on the work of Sochen-Kimmel-Malladi [20], [21]. 


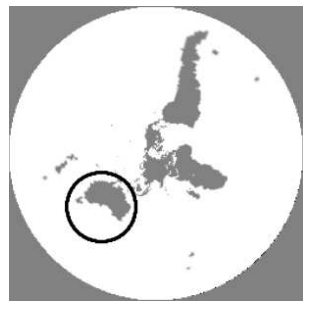

(a) Initial $\mathrm{AC}$ on

D

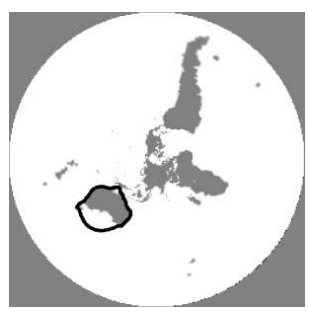

(c) AC at $t_{2}>t_{1}$

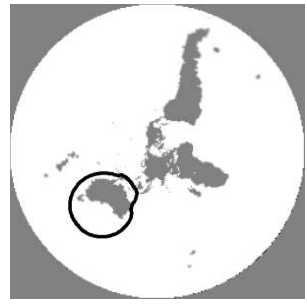

(b) AC at $t_{1}>0$

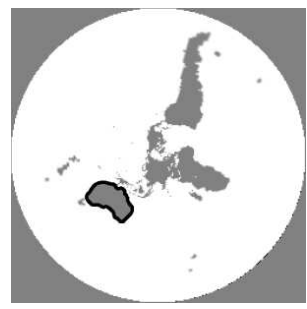

(d) Final AC

Fig. 11. Segmentation of Australia on the Sphere. Figures (a-d) present the evolution of the AC on the disk $D$ given by Equation (36). The disk $D$ corresponds to the stereographic projection of the sphere onto the plane.

We consider the following functional introduced in [19], [3]:

$$
\left\{\begin{array}{l}
P_{f}(X, \Sigma, \Omega)= \\
\quad \int d^{n_{\Sigma}} \varsigma f\left(X, \sigma_{\mu \nu}, h_{i j}\right) \sigma^{1 / 2} \sigma^{\mu \nu} \partial_{\mu} X^{i} \partial_{\nu} X^{j} h_{i j}, \\
X:\left(\Sigma, \sigma_{\mu \nu}\right) \rightarrow\left(\Omega, h_{i j}\right),
\end{array}\right.
$$

which corresponds to the Polyakov action [22] weighted by the function $f$. The Polyakov action is basically a functional that measures the weight of a mapping $X$ between an embedded manifold $\Sigma$ and the embedding manifold $\Omega$ (see Figure 13).

More precisely, $\sigma_{\mu \nu}$ is the first fundamental form of the manifold $\Sigma, \sigma^{\mu \nu}$ is the inverse metric of $\sigma_{\mu \nu}, \sigma$ is the determinant of $\sigma_{\mu \nu}, n_{\Sigma}$ is the dimension of $\Sigma, \mu, \nu=1, \ldots, n_{\Sigma}, d^{n_{\Sigma}} \varsigma \sigma^{1 / 2}$ is the volume element w.r.t. the local coordinates on $\Sigma, h_{i j}$ is the metric tensor of the embedding space $\Omega, n_{\Omega}$ the dimension of $\Omega, i, j=1, \ldots, n_{\Omega}, \partial_{\mu} X^{i}=\partial X^{i} / \partial \varsigma^{\mu}$ and $\sigma^{\mu \nu} \partial_{\mu} X^{i} \partial_{\nu} X^{j} h_{i j}$ is the generalization of the magnitude of the gradient to maps between Riemannian manifolds. We observe that the volume element as well as the rest of the expression is re-parametrization invariant. In other words, they are invariant 


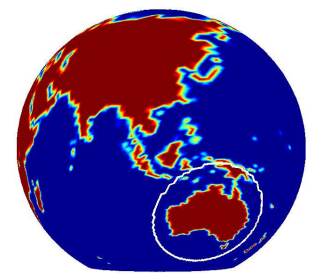

(a) Initial $\mathrm{AC}$ on $S^{2}$

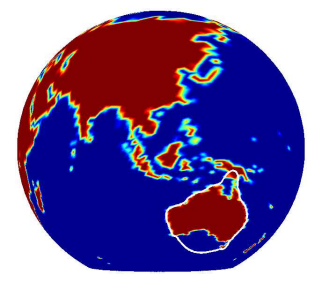

(c) AC at $t_{2}>t_{1}$

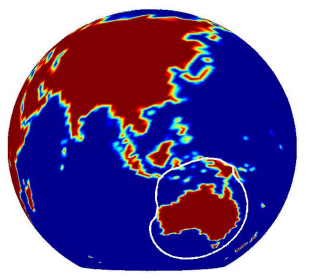

(b) $\mathrm{AC}$ at $t_{1}>0$

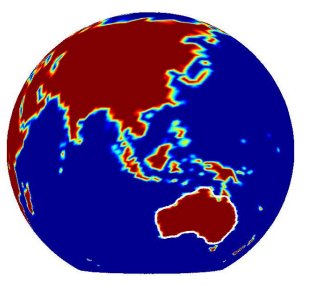

(d) Final AC

Fig. 12. Segmentation of Australia on the Sphere. Figures (a-d) show the evolution of the AC of Figure 11 on the sphere $S^{2}$ using the mapping from the disk to the sphere defi ned in Equation (40).

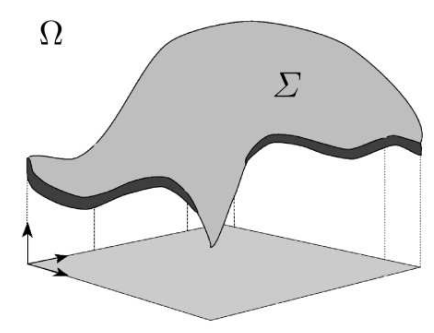

Fig. 13. The manifold $\Sigma$ embedded in $\Omega$, reproduced from [20].

under a smooth transformation. Thus, this action depends on the geometrical objects and not on the way we describe them via our parametrization of the coordinates. Finally, when identical indices appear one up and one down in Equation (42), they are summed over according to the Einstein summation convention.

The calculus of variations gives us the flow minimizing Functional (42) w.r.t. the $l$-th embedding 
coordinate $X^{l}, \sigma_{\mu \nu}$ and $h_{i j}$ being fixed:

$$
\begin{aligned}
\frac{\partial X^{l}}{\partial t}= & f\left(X, \sigma_{\mu \nu}, h_{i j}\right) \cdot\left(\sigma^{-1 / 2} \partial_{\mu}\left(\sigma^{1 / 2} \sigma^{\mu \nu} \partial_{\nu} X^{l}\right)+\right. \\
& \left.\Gamma_{j k}^{l} \partial_{\mu} X^{j} \partial_{\nu} X^{k} \sigma^{\mu \nu}\right)+\partial_{k} f \sigma^{\mu \nu} \partial_{\mu} X^{k} \partial_{\nu} X^{l} \\
& -\frac{n_{\Omega}}{2} h^{l k} \partial_{k} f \sigma^{\mu \nu} \partial_{\mu} X^{i} \partial_{\nu} X^{j} h_{i j},
\end{aligned}
$$

for $1 \leq l \leq n_{\Omega}, g^{-1 / 2} \partial_{\mu}\left(\sigma^{1 / 2} \sigma^{\mu \nu} \partial_{\nu} X^{l}\right)$ is the Beltrami operator which generalizes the Laplace operator to non-flat manifolds and $\Gamma_{j k}^{l}=\frac{1}{2} h^{l i}\left(\partial_{j} h_{i k}+\partial_{k} h_{j i}-\partial_{i} h_{j k}\right)$ is the Levi-Civita connection coeffi cients. If the metric tensor $\sigma_{\mu \nu}$ of the embedded manifold $\Sigma$ is chosen to be the induced metric tensor; $\sigma_{\mu \nu}=$ $\partial_{\mu} X^{i} \partial_{\nu} X^{j} h_{i j}$, then the map $X$ are harmonic maps such as geodesics and minimal surfaces and the weighted Polyakov action is reduced to the weighted Euler functional/Nambu action that describes the (hyper-)area of a (hyper-)surface $\Sigma$ :

$$
S_{f}=\int d^{n_{\Sigma}} \varsigma f \sigma^{1 / 2}
$$

The induced metric tensor is also introduced in the flow (43), which yields to:

$$
\left\{\begin{aligned}
& \frac{\partial X^{l}}{\partial t}= f \cdot \mathcal{H}^{l}+\partial_{k} f \sigma^{\mu \nu} \partial_{\mu} X^{k} \partial_{\nu} X^{l}-\frac{n_{\Omega} \cdot n_{\Sigma}}{2} \partial_{k} f h^{k l} \\
& \mathcal{H}^{l}=\left(\sigma^{-1 / 2} \partial_{\mu}\left(\sigma^{1 / 2} \sigma^{\mu \nu} \partial_{\nu} X^{l}\right)+\right. \\
&\left.\Gamma_{j k}^{l} \partial_{\mu} X^{j} \partial_{\nu} X^{k} \sigma^{\mu \nu}\right)_{\sigma_{\mu \nu}=\partial_{\mu} X^{i} \partial_{\nu} X^{j} h_{i j}}
\end{aligned}\right.
$$

for $1 \leq l \leq n_{\Omega}$ and $\mathcal{H}$ is the mean curvature vector generalized to any embedding manifold $\Omega$. Thus the functional (44) and the minimization flow (45) defi ne the energy and the evolution equation for the active contour model defi ned in a general Riemannian manifold such as hyperbolic, spherical or parabolic manifolds.

The previous model is consistent with the standard geodesic/geometric active contours model [2], [16] when the embedding manifold is the Euclidean space, i.e. $\Omega=\mathbb{R}^{2}, h_{i j}=\delta_{i j}$. Indeed, let us choose $X=C: p \rightarrow\left(C^{1}(p), C^{2}(p)\right)$, which means that the metric tensor of $\Sigma$ is as follows:

$$
\begin{aligned}
\sigma_{\mu \nu}=\partial_{\mu} X^{i} \partial_{\nu} X^{j} h_{i j} & =\partial_{\mu} C^{i} \partial_{\nu} C^{j} \delta_{i j} \\
& =\left|C_{p}\right|^{2}=\sigma_{p p}=\sigma
\end{aligned}
$$

with $\mu=\nu=p$. Energy functional (44) is thus equal to

$$
\int d^{n_{\Sigma}} \varsigma f \sigma^{1 / 2}=\int d p f\left|C_{p}\right|=F_{G A C}(C)
$$


which corresponds to the energy of the geodesic/geometric active contour model defi ned in Equation (28) when $f$ is the edge detector function. The minimization flow (29) can also be recovered. The mean curvature vector is equal to

$$
\begin{aligned}
& \mathcal{H}^{l}=\left(\sigma^{-1 / 2} \partial_{\mu}\left(\sigma^{1 / 2} \sigma^{\mu \nu} \partial_{\nu} X^{l}\right)+\right. \\
&\left.\Gamma_{j k}^{l} \partial_{\mu} X^{j} \partial_{\nu} X^{k} \sigma^{\mu \nu}\right)_{\sigma_{\mu \nu}=\partial_{\mu} X^{i} \partial_{\nu} X^{j} h_{i j}} \\
&=\frac{1}{\left|C_{p}\right|} \partial_{p}\left(\frac{\partial_{p} C^{l}}{\left|C_{p}\right|}\right)=\frac{1}{\left|C_{p}\right|} \partial_{p} \mathcal{T}^{l}=\kappa \mathcal{N}^{l},
\end{aligned}
$$

using $\mathcal{T}=C_{p} /\left|C_{p}\right|$ and $\partial_{p} \mathcal{T}=\left|C_{p}\right| \partial_{s} \mathcal{T}=\left|C_{p}\right| \kappa \mathcal{N}$. The second part of the flow is equal to:

$$
\begin{aligned}
& \partial_{k} f \sigma^{\mu \nu} \partial_{\mu} X^{k} \partial_{\nu} X^{l}-\frac{n_{\Omega} \cdot n_{\Sigma}}{2} \partial_{k} f h^{k l} \\
= & \frac{1}{\left|C_{p}\right|^{2}} \partial_{k} f \cdot \partial_{p} C^{k} \partial_{p} C^{l}-\partial_{k} f \delta^{k l} \\
=\left\langle\nabla f, \frac{C_{p}}{\left|C_{p}\right|}\right\rangle \frac{C_{p}^{l}}{\left|C_{p}\right|}-\partial_{l} f & =\langle\nabla f, \mathcal{T}\rangle \mathcal{T}^{l}-\partial_{l} f \\
= & -\left\langle\nabla f, \mathcal{N}^{l}\right\rangle \mathcal{N}^{l} .
\end{aligned}
$$

Finally, the flow (45) is as follows:

$$
\partial_{t} C=f \kappa \mathcal{N}-\langle\nabla f, \mathcal{N}\rangle \mathcal{N}
$$

which is exactly the flow of the geodesic/geometric active contour model defi ned in Equation (29).

We now determine the energy and the evolution equation for the active contours evolving on parabolic manifolds. We remind the metric tensor for a parabolic manifold, defi ned in Equation (8):

$$
\left(h_{i j}\right)_{P^{2}}=\left(\begin{array}{cc}
1+4 x^{2} & 4 x y \\
4 x y & 1+4 y^{2}
\end{array}\right),
$$

and the inverse tensor is as follows:

$$
\left(h^{i j}\right)_{P^{2}}=h^{-1}\left(\begin{array}{cc}
1+4 y^{2} & -4 x y \\
-4 x y & 1+4 x^{2}
\end{array}\right),
$$

where $h=1+4 x^{2}+4 y^{2}$ is the determinant of $h_{i j}$. The metric tensor of the active contour, called $\Sigma$ in 
the Polyakov framework, embedded on a parabolic manifold, called $\Omega$, is as follows:

$$
\begin{aligned}
& \sigma_{\mu \nu}= \partial_{\mu} X^{i} \partial_{\nu} X^{j} h_{i j}=\partial_{p} C^{i} \partial_{p} C^{j} h_{i j} \\
&=\left|C_{p}\right|^{2}\left(\frac{C_{p}^{1}}{\left|C_{p}\right|} \frac{C_{p}^{1}}{\left|C_{p}\right|} h_{x x}+2 \frac{C_{p}^{1}}{\left|C_{p}\right|} \frac{C_{p}^{2}}{\left|C_{p}\right|} h_{x y}+\right. \\
&\left.\frac{C_{p}^{2}}{\left|C_{p}\right|} \frac{C_{p}^{2}}{\left|C_{p}\right|} h_{y y}\right) \\
&=\left|C_{p}\right|^{2}\left(\mathcal{T}^{1} \mathcal{T}^{1} h_{x x}+2 \mathcal{T}^{1} \mathcal{T}^{2} h_{x y}+\mathcal{T}^{2} \mathcal{T}^{2} h_{y y}\right) \\
&=\left|C_{p}\right|^{2} \mathcal{T}^{T}\left(h_{i j}\right)_{P^{2}} \mathcal{T}=\left|C_{p}\right|^{2}|\mathcal{T}|_{\left(h_{i j}\right)_{P^{2}}}=\sigma_{p p} \\
&=\sigma
\end{aligned}
$$

where $\mathcal{T}^{T}$ means the transpose of $\mathcal{T}$ and $|\mathcal{T}|_{\left(h_{i j}\right)_{P^{2}}}$ is the norm of the tangent vector on the parabolic manifold. Thus, Energy functional (44) of the active contours embedded on a parabolic manifold is equal to

$$
\begin{aligned}
\int d^{n_{\Sigma}} \varsigma f \sigma^{1 / 2} & =\int f|\mathcal{T}|_{\left(h_{i j}\right)_{P^{2}}}\left|C_{p}\right| d p \\
& =\int f|\mathcal{T}|_{\left(h_{i j}\right)_{P^{2}}} d s=F_{A C P M}(C),
\end{aligned}
$$

where $A C P M$ stands for Active Contours on Parabolic Manifolds. Let us now compute the evolution equation for the active contours on a parabolic manifold. The Beltrami part is equal to:

$$
\begin{array}{r}
\sigma^{-1 / 2} \partial_{\mu}\left(\sigma^{1 / 2} \sigma^{\mu \nu} \partial_{\nu} X^{l}\right)=\sigma^{-1 / 2} \partial_{p}\left(\sigma^{-1 / 2}\left|C_{p}\right| \frac{\partial_{p} C^{l}}{\left|C_{p}\right|}\right) \\
=\sigma^{-1}\left|C_{p}\right|^{2} \kappa \mathcal{N}^{l}-\frac{1}{2} \sigma^{-2}\left|C_{p}\right|^{2}\langle\nabla \sigma, \mathcal{T}\rangle \mathcal{T}^{l}
\end{array}
$$

The Levi-Civita connection coeffi cients are equal to:

$$
\begin{aligned}
\Gamma_{j k}^{l} & =\frac{1}{2} h^{l i}\left(\partial_{j} h_{i k}+\partial_{k} h_{j i}-\partial_{i} h_{j k}\right), \\
\Gamma_{j k}^{1} & =4 h^{-1} x \delta_{j k} \\
\Gamma_{j k}^{2} & =4 h^{-1} y \delta_{j k},
\end{aligned}
$$


which gives us the second term of the mean curvature vector:

$$
\begin{aligned}
\Gamma_{j k}^{1} \partial_{p} C^{j} \partial_{p} C^{k} \sigma^{p p} & =4 h^{-1} x \delta_{j k} \partial_{p} C^{j} \partial_{p} C^{k} \sigma^{-1}, \\
& =4 h^{-1} \sigma^{-1}\left|C_{p}\right|^{2} x, \\
\Gamma_{j k}^{2} \partial_{p} C^{j} \partial_{p} C^{k} \sigma^{p p} & =4 h^{-1} y \delta_{j k} \partial_{p} C^{j} \partial_{p} C^{k} \sigma^{-1}, \\
& =4 h^{-1} \sigma^{-1}\left|C_{p}\right|^{2} y,
\end{aligned}
$$

which is as follows under the vectorial form:

$$
\Gamma_{j k} \partial_{\mu} X^{j} \partial_{\nu} X^{k} \sigma^{\mu \nu}=4 h^{-1} \sigma^{-1}\left|C_{p}\right|^{2} \mathbf{x}
$$

where $\mathbf{x}=(x, y)$. Thus, the mean curvature vector is equal to:

$$
\begin{aligned}
& \mathcal{H}=\left(\sigma^{-1 / 2} \partial_{\mu}\left(\sigma^{1 / 2} \sigma^{\mu \nu} \partial_{\nu} X\right)+\right. \\
&\left.\Gamma_{j k} \partial_{\mu} X^{j} \partial_{\nu} X^{k} \sigma^{\mu \nu}\right)_{\sigma_{\mu \nu}=\partial_{\mu} X^{i} \partial_{\nu} X^{j} h_{i j}} \\
&=\sigma^{-1}\left|C_{p}\right|^{2} \kappa \mathcal{N}-\frac{1}{2} \sigma^{-2}\left|C_{p}\right|^{2}\langle\nabla \sigma, \mathcal{T}\rangle \mathcal{T} \\
& \\
&+4 h^{-1} \sigma^{-1}\left|C_{p}\right|^{2} \mathbf{x}
\end{aligned}
$$

Then, the second part of the flow (45) is equal to:

$$
\begin{aligned}
\partial_{k} f \sigma^{\mu \nu} \partial_{\mu} X^{k} \partial_{\nu} X^{l} & =\partial_{k} f \sigma^{p p} \partial_{p} C^{k} \partial_{p} C^{l} \\
& =\sigma^{-1}\left|C_{p}\right|^{2}\langle\nabla f, \mathcal{T}\rangle \mathcal{T}^{l}
\end{aligned}
$$

and the term $-\frac{n_{\Omega} \cdot n_{\Sigma}}{2} \partial_{k} f h^{k l}$ is equal for $l=1,2$ to:

$$
\begin{aligned}
& -\left(\begin{array}{c}
\partial_{x} f h^{x x}+\partial_{y} f h^{x y} \\
\partial_{x} f h^{x y}+\partial_{y} f h^{y y}
\end{array}\right)= \\
& -\left(\begin{array}{cc}
h^{x x} & h^{x y} \\
h^{x y} & h^{y y}
\end{array}\right)\left(\begin{array}{c}
\partial_{x} f \\
\partial_{y} f
\end{array}\right)=-\left(h^{i j}\right) \nabla f=-\nabla^{\Omega} f,
\end{aligned}
$$

where $\nabla^{\Omega}$ is the gradient operator defi ned on the manifold $\Omega$. Finally, the flow (45) for the active contours embedded on a parabolic manifold is a follows:

$$
\begin{aligned}
\partial_{t} C= & f \cdot\left[\sigma^{-1}\left|C_{p}\right|^{2} \kappa \mathcal{N}-\frac{1}{2} \sigma^{-2}\left|C_{p}\right|^{2}\langle\nabla \sigma, \mathcal{T}\rangle \mathcal{T}+\right. \\
& \left.4 h^{-1} \sigma^{-1}\left|C_{p}\right|^{2} \mathbf{x}\right]+\sigma^{-1}\left|C_{p}\right|^{2}\langle\nabla f, \mathcal{T}\rangle \mathcal{T}-\nabla^{\Omega} f .
\end{aligned}
$$


In [23], Epstein-Gage proved that the geometry of the curve deformation is not affected by the tangential velocity $\mathcal{T}$. This result is due to the fact that the tangential velocity does not change the geometry of the curve but its parametrization. Hence, Equation (76) can be replaced by

$$
\begin{gathered}
\partial_{t} C=\sigma^{-1}\left|C_{p}\right|^{2}\left(f \kappa+4 h^{-1}\langle\mathbf{x}, \mathcal{N}\rangle\right) \mathcal{N} \\
-\left\langle\nabla^{\Omega} f, \mathcal{N}\right\rangle \mathcal{N}
\end{gathered}
$$

Since the minimization solution is not changed when the Euler-Lagrange equation is multipliedby a strictly positive function, we fi nally get the evolution of the active contour on a parabolic manifold:

$$
\partial_{t} C=\left(f \kappa-|\mathcal{T}|_{\left(h_{i j}\right)_{P^{2}}}\left\langle\nabla^{\Omega} f, \mathcal{N}\right\rangle+4 h^{-1}\langle\mathbf{x}, \mathcal{N}\rangle\right) \mathcal{N}
$$

using $\sigma\left|C_{p}\right|^{-2}=|\mathcal{T}|_{\left(h_{i j}\right)_{P}^{2}}$.

As an example of illustration, we consider the segmentation of the bunny object lying on the parabolic manifold on Figures 15 and 16. The mapping between the disk $D$ on Figure 14(a) and the paraboloid, which corresponds to the focal projection of the paraboloid onto the plane, is given by:

$$
\begin{aligned}
(x, y) \in D \rightarrow & \left(x_{P^{2}}, y_{P^{2}}, z_{P^{2}}\right)= \\
& \left(r \cos \varphi, r \sin \varphi, r^{2}\right) \in P^{2},
\end{aligned}
$$

given

$$
(r, \varphi)=\left(\sqrt{x^{2}+y^{2}}, \tan ^{-1}\left(\frac{y}{x}\right)\right) .
$$

The numerical implementation of the minimizing flow (78) is done with classical numerical schemes, see [17], [18] for details.

\section{Why Using Manifold's Geometry in the Active Contour Evolution Process?}

Previous sections defi ned evolution equations for active contours evolving on different manifolds. We remind that our basic idea consists in evolving the snake on the disk using the geometry of the given manifold and map the evolving contour on the manifold. But is it really useful to include the manifold's geometry in the evolution process? In other words, why not just using the standard Euclidean active contour model [2], [16] on the disk and map the evolving contour to the manifold?

We introduce here an example to show the importance of taking into account the geometry of the given manifold in the segmentation process. Figure 17(a) shows an object of interest defi ned on the hyperbolic manifold. This object presents a smooth transition on the hyperboloid manifold, as one can see on Figure 


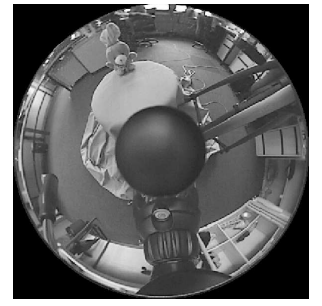

(a) Bunny Image on the Disk $D$

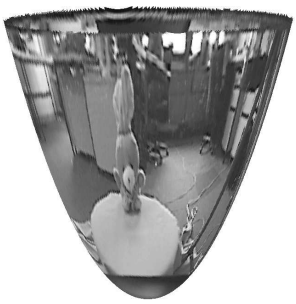

(b) Bunny Image on the Paraboloid $P^{2}$

Fig. 14. The original bunny image obtained from a catadioptric system. (a) Raw image from the sensor. (b) Omnidirectional image represented as a scalar function on the paraboloid.

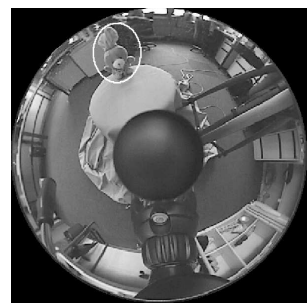

(a) Initial $\mathrm{AC}$ on

D

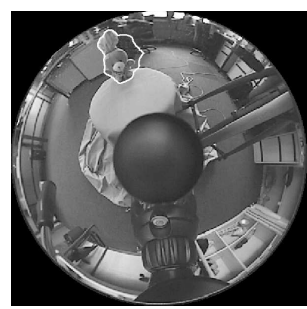

(c) $\mathrm{AC}$ at $t_{2}>t_{1}$

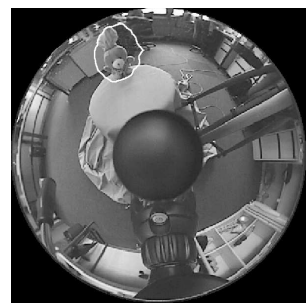

(b) $\mathrm{AC}$ at $t_{1}>0$

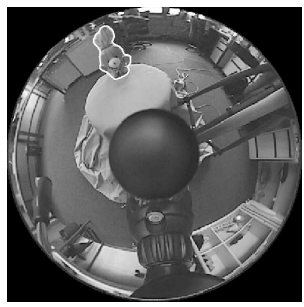

(d) Final AC

Fig. 15. Segmentation of Bunny on the Paraboloid. Figures (a-d) present the evolution of the AC on the disk $D$ given by Equation (78). The disk $D$ corresponds to the focal projection of the paraboloid onto the plane.

17(b), which obviously does not correspond to an edge. However, the projection of this smooth part on the plane, Figure 17(c), shows a sharp transition corresponding to an edge on the plane. This false 'edge' can give unsatisfactory segmentation results if the hyperboloid geometry is not considered. Results based 


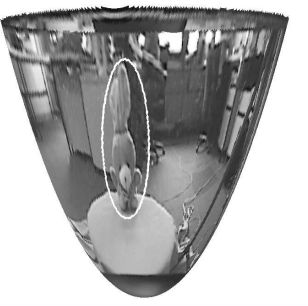

(a) Initial $\mathrm{AC}$ on $S^{2}$

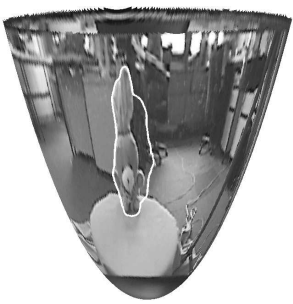

(c) AC at $t_{2}>t_{1}$

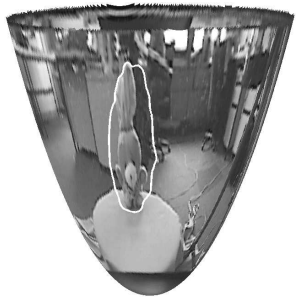

(b) AC at $t_{1}>0$

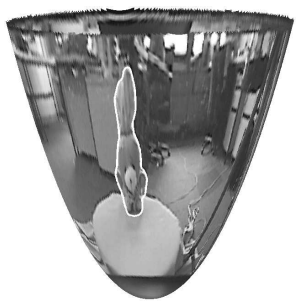

(d) Final AC

Fig. 16. Segmentation of Bunny on the Paraboloid. Figures (h-k) show the evolution of the AC of Figure 15 on the paraboloid $P^{2}$ using the mapping from the disk to the paraboloid defi ned in Equation (79).

on the Euclidean geometry and the hyperbolic geometry are presented on Figures 18 and 19. Figures 18(a-h) show the Euclidean active contour which fails to segment the whole object on the hyperboloid due to the false edge on the disk that corresponds to a smooth transition on the hyperboloid. Figures 18(i-p) present the hyperbolic active contour that succeeds capturing the entire object because the snake takes into account the geometry of the hyperboloid manifold, which multiplies the edge detector function by a factor $\frac{\left|1-r^{2}\right|}{2}$, in its evolution.

\section{Discussion with Related Works}

As we said in Section III, Bertalmio et al. in [12], [13] introduced a new framework for solving PDEs on arbitrary surfaces implicitly represented by a level set function. The main advantage of their approach is to deal with arbitrary surfaces, which is not our case, even if a data extension process is needed and the computational time is more important than our technique. In our framework, surfaces are given by a global parametrization, which allows accurate and fast computations of the active contour flows.

In [24], [25], Spira and Kimmel defi ned the evolution equation of the geodesic active contour on 


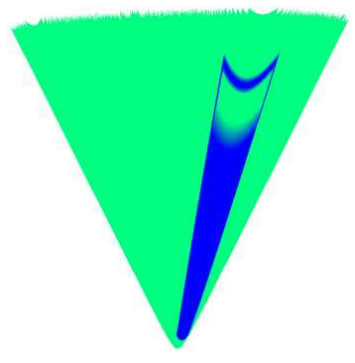

(a) Original image on the Hyperboloid.

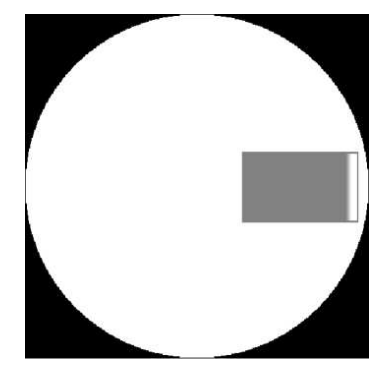

(c) Original Image on the Disk.

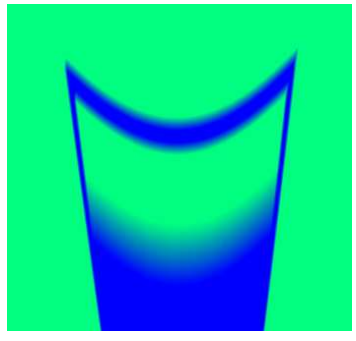

(b) Zoom on the Object of Interest.

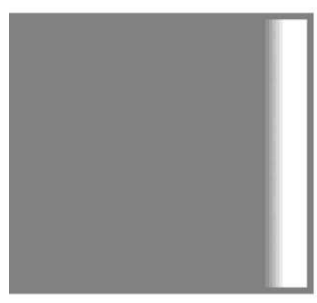

(d) Zoom on the Object of Interest.

Fig. 17. Figures (a-b) present an object of interest, composed of a smooth transition, painted on the hyperbolic manifold. Figures (c-d) show the projection of the object on the plane. The smooth part of the signal on the hyperboloid corresponds to a sharp transition on the plane.

global parametric manifolds by considering the Riemannian counterparts of the Euclidean flow [2]. Our approach is closely related to theirs because we also consider parametric manifolds. However, the main difference lies in the fact that we have developed the flow for the active contours defi ned on parametric manifolds from a variational model based on the weighted Polyakov functional, which gives the energy of the active contours and the weighted Riemannian length of contours.

\section{CONCLusions}

Omnidirectional imaging holds interesting promises for applications and will certainly become a conventional technique in the near future. Due to their (eventually near) full covering of the field of view, omnidirectional images allow to monitor a complete scene using a single frame and thus provide a very appealing basis for many computer vision algorithms. Manufacturing perfect omnidirectional image 


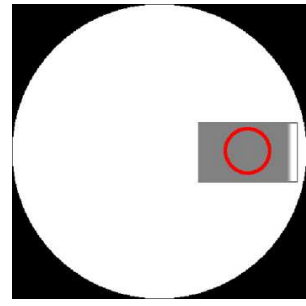

(a) Euclidean $\mathrm{AC}$ on the Disk at $t_{1}=0$.

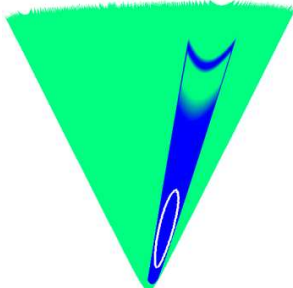

(e) Euclidean $\mathrm{AC}$ on the Hyperboloid at $t_{1}=0$.
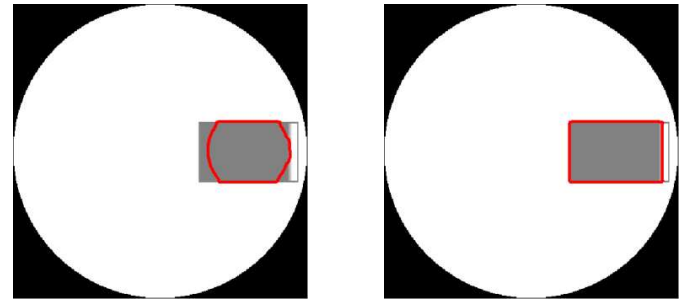

(b) Euclidean AC at $t_{2}>t_{1}$.

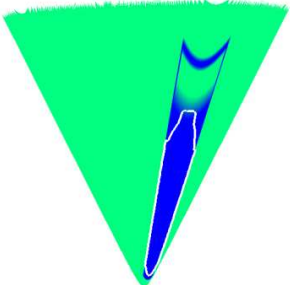

(f) Euclidean $\mathrm{AC}$ at $t_{2}>t_{1}$. (c) Euclidean AC at $t_{3}>t_{2}$.

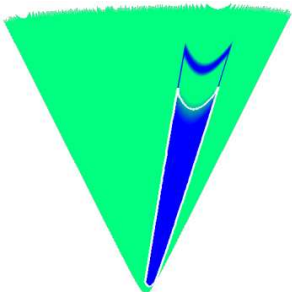

(g) Euclidean AC at $t_{3}>t_{2}$.

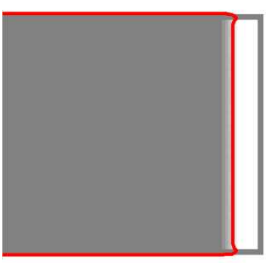

(d) Zoom on the $\mathrm{AC}$ at $t_{3}$.

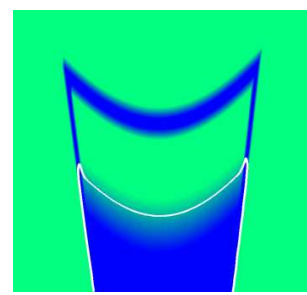

(h) Zoom on the $\mathrm{AC}$ at $t_{3}$.

Fig. 18. Segmentation of a synthetic object on the hyperboloid with the standard active contour model. The standard Euclidean active contour (AC) fails to segment the whole object on the hyperboloid due to a false edge on the disk, Figures (a-d), corresponding to a smooth transition on the hyperboloid, Figures (e-h).

acquisition systems seems to be very challenging, but several groups have reported interesting progress [5]. A very interesting and promising research direction is to investigate the properties of insect or animal eyes to design more effective optical devices [26], [27]. Before a new generation of full fi eld of view optical devices matures, the conventional way of acquiring omnidirectional images is through a standard catadioptric setting, where a standard sensor overlooks a curved mirror. The images obtained by such a device are strongly influenced by the geometry of the mirror.

In this paper, we showed that the output of a catadioptric system can be seen as a scalar function on a special riemannian surface whose metric can be explicitly computed from parameters of the system. We showed that it is important to take these geometric effects into account when using such images for computer vision and methods based on Partial Differential Equations offer the necessary flexibility. 


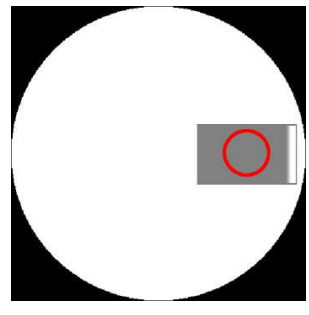

(a) Hyperbolic $\mathrm{AC}$ on the Disk at $t_{1}=0$.

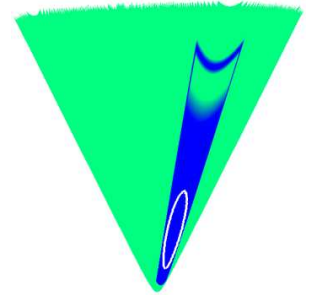

(e) Hyperbolic

$\mathrm{AC}$ on the

Hyperboloid at

$t_{1}=0$.
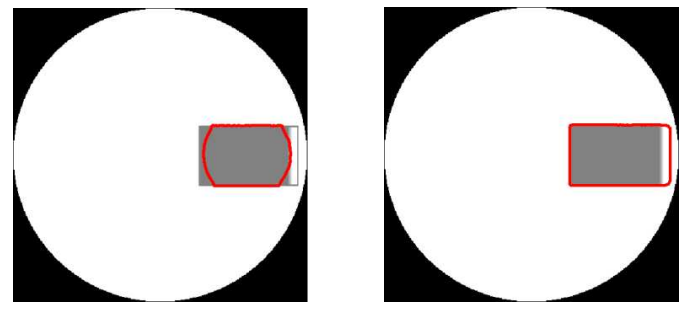

(b) Hyperbolic

$\mathrm{AC}$ at $t_{2}>t_{1}$.

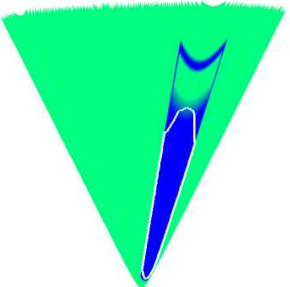

(f) Hyperbolic

$\mathrm{AC}$ at $t_{2}>t_{1}$. (c) Hyperbolic

$\mathrm{AC}$ at $t_{3}>t_{2}$.

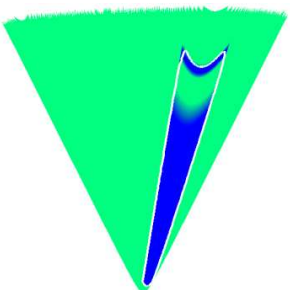

(g) Hyperbolic

$\mathrm{AC}$ at $t_{3}>t_{2}$.

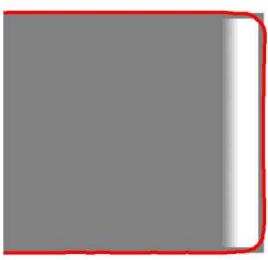

(d) Zoom on the $\mathrm{AC}$ at $t_{3}$.

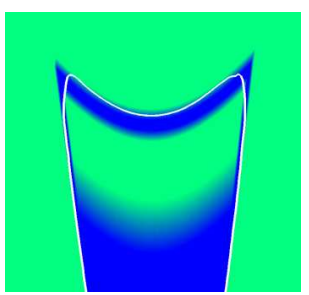

(h) Zoom on the $\mathrm{AC}$ at $t_{3}$.

Fig. 19. Segmentation of a synthetic object on the hyperboloid with the proposed active contour model. The hyperbolic active contour (AC) succeeds capturing the entire object, whereas the Euclidean model failed on Figure 18, because the proposed AC takes into account the geometry of the hyperbolic manifold in its evolution.

First, we derived explicit expressions for standard differential operators used for smoothing or edge detection. Then, we introduced new energy functionals suited for solving segmentation problems on catadioptric images and solved for curve evolution equations using standard calculus of variations. The obtained geometric active contours are appealing because they are able to deal with some of the apparent distortions that plague omnidirectional images. Moreover, for the class of riemannian surfaces studied in this paper, computations involve only standard euclidean fi nite differences on the image plane, which means we work directly with sensor pixels.

In a more general perspective, performing smoothing or solving segmentation problems on arbitrary manifolds is a challenge of signifi cant importance for processing large higher-dimensional data sets. For example, emerging bioimaging techniques easily acquire 3D time varying images that need to be 
automatically segmented [28]. Extending the framework based on the Polyakov action introduced in Section IV could be an interesting way to leverage our results to a much more general case and will be the main topic of a forthcoming paper.

\section{REFERENCES}

[1] C. Geyer and K. Daniilidis, "Catadioptric projective geometry," Internationa Journal of Computer Vision, vol. 45(3), pp. 223-243, 2001.

[2] V. Caselles, R. Kimmel, and G. Sapiro, “Geodesic Active Contours," International Journal of Computer Vision, vol. 22(1), pp. 61-79, 1997.

[3] X. Bresson, P. Vandergheynst, and J.-P. Thiran, "Multiscale Active Contours," International Journal of Computer Vision, vol. 70(3), pp. 197-211, 2006.

[4] M. Do Carmo, Riemannian Geometry. Birkhauser, 1992.

[5] J. Neumann, C. Fermüller, and Y. Aloimonos, "Eyes from eyes: New cameras for structure from motion," in Third Workshop on Omnidirectional Vision, June 2002, pp. 19-26.

[6] S. Rosenberg, The Laplacian on a Riemannian manifold. Cambridge University Press, 1997.

[7] L. Florack, B. ter Haar Romeny, J. Koenderink, and A. Viergever, "Linear scale-space," Journal of Mathematical Imaging and Vision, vol. 4, no. 4, pp. 225-251, 1994.

[8] A. P. Witkin, "Scale-space fi ltering," in Proc. Int. Joint. Conf. Artificial Intelligence, 1983, pp. 1019-1021.

[9] T. Lindeberg, Scale-Space Theory in Computer Vision. Netherlands: Kluwer Academic Publishers, 1994.

[10] D. Healy, D. Rockmore, P. Kostelec, and S. Moore, "Ffts for the 2-sphere - improvements and variations," J. Fourier Anal. Appl., vol. 9, no. 4, pp. $341-385,2003$.

[11] I. Bogdanova, P. Vandergheynst, and J.-P. Gazeau, "Continuous wavelet transform on the hyperboloid," submitted to Applied and Computational Harmonic Analysis, June 2005.

[12] M. Bertalmío, L.-T. Cheng, S. Osher, and G. Sapiro, "Variational Problems and Partial Differential Equations on Implicit Surfaces,” Journal of Computational Physics, vol. 174(2), pp. 759-780, 2001.

[13] M. Bertalmío, F. Mémoli, , L.-T. Cheng, G. Sapiro, and S. Osher, "'”Variational Problems and Partial Differential Equations on Implicit Surfaces: Bye Bye Triangulated Surfaces?” in Geometric Level Set Methods in Imaging, Vision and Graphics," eds. S. Osher and N. Paragios, Springer-Verlag, NY, pp. 381-397, 2003.

[14] S. Chen, B. Merriman, S. Osher, and P. Smereka, “A Simple Level Set Method for Solving Stefan Problems,” Journal of Computational Physics, vol. 135(1), pp. 8-29, 1997.

[15] G. Aubert and P. Kornprobst, Mathematical Problems in Image Processing. New York: Springer, 2002.

[16] S. Kichenassamy, A. Kumar, P. Olver, A. Tannenbaum, and A. Yezzi, "Conformal Curvature Flows: From Phase Transitions to Active Vision," Archive for Rational Mechanics and Analysis, vol. 134, pp. 275-301, 1996.

[17] S. Osher, "'Level Set Methods", in Geometric Level Set Methods in Imaging, Vision and Graphics," eds. S. Osher and N. Paragios, Springer-Verlag, NY, pp. 3-20, 2003.

[18] J. A. Sethian, Level Set Methods and Fast Marching Methods: Evolving Interfaces in Computational Geometry, Fluid Mechanics, Computer Vision and Matherial Sciencies. Cambridge: University Press, 1999.

[19] X. Bresson, P. Vandergheynst, and J.-P. Thiran, "Multiscale Active Contours," in Proceedings of 5th International Conference on Scale Space and PDE methods in Computer Vision, 2005, pp. 167-178. 
[20] N. Sochen, R. Kimmel, and R. Malladi, “A General Framework for Low Level Vision,” IEEE Transactions on Image Processing, vol. 7(3), pp. 310 - 318, 1998.

[21] _ _ "A General Framework for Low Level Vision, Technical Report LBNL-39243, Physics Department, Berkeley,” 1996.

[22] A. M. Polyakov, "Quantum Geometry of Bosonic Strings," Physics Letters B, vol. 103, pp. 207-210, 1981.

[23] C. L. Epstein and M. Gage, "The Curve Shortening Flow," in Wave Motion: Theory, Modeling, and Computation, A. Chorin and A. Majda, Editors, Springer-Verlag, New York, vol. MR 89f:58128, 1987.

[24] A. Spira and R. Kimmel, "Geodesic Curvature Flow on Parametric Surfaces," in Curve and Surface Design: Saint-Malo 2002, 2002, pp. 365-373.

[25] — - "Segmentation of Images Painted on Parametric Manifolds," in EUSIPCO, 2005.

[26] National Geographic News, "Animal eyes provide high-tech optical inspiration,” December 2005.

[27] R. Zbikowski, "Fly like a fly," IEEE Spectrum, vol. 42, no. 11, pp. 40-45, November 2005.

[28] J. Kovacevic and R. Murphy, "Molecular and cellular bioimaging," IEEE Signal Processing Magazine, guest editorial, vol. 23 , no. 3 , p. 19, 2006. 